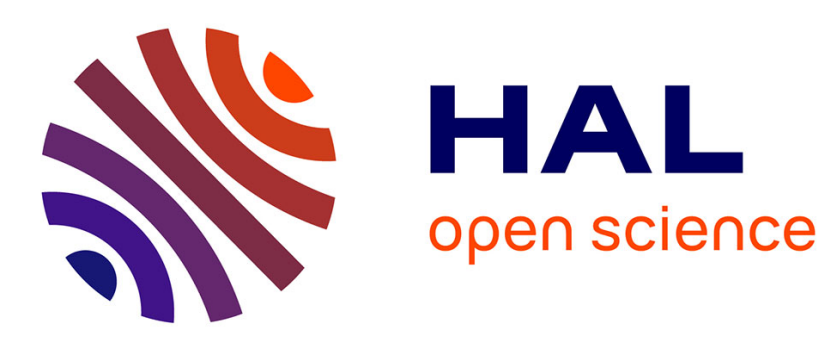

\title{
Optimal Solution of the Maximum All Request Path Grooming Problem
}

Jean-Claude Bermond, Michel Cosnard, David Coudert, Stéphane Pérennes

\section{To cite this version:}

Jean-Claude Bermond, Michel Cosnard, David Coudert, Stéphane Pérennes. Optimal Solution of the Maximum All Request Path Grooming Problem. [Research Report] RR-5627, INRIA. 2006, pp.12. inria-00070381

\section{HAL Id: inria-00070381 https://hal.inria.fr/inria-00070381}

Submitted on 19 May 2006

HAL is a multi-disciplinary open access archive for the deposit and dissemination of scientific research documents, whether they are published or not. The documents may come from teaching and research institutions in France or abroad, or from public or private research centers.
L'archive ouverte pluridisciplinaire HAL, est destinée au dépôt et à la diffusion de documents scientifiques de niveau recherche, publiés ou non, émanant des établissements d'enseignement et de recherche français ou étrangers, des laboratoires publics ou privés. 


\title{
Optimal Solution of the Maximum All Request Path Grooming Problem
}

\author{
Jean-Claude Bermond — Michel Cosnard — David Coudert — Stéphane Pérennes
}

$\mathbf{N}^{\circ} 5627$

Juillet 2005

Thème COM 



\title{
Optimal Solution of the Maximum All Request Path Grooming Problem
}

\author{
Jean-Claude Bermond, Michel Cosnard, David Coudert, Stéphane Pérennes \\ Thème COM - Systèmes communicants \\ Projet Mascotte, commun I3S-CNRS/INRIA/UNSA \\ Rapport de recherche $\mathrm{n}^{\circ} 5627$ - Juillet 2005 - 12 pages
}

\begin{abstract}
We give an optimal solution to the Maximum All Request Path Grooming (MARPG) problem motivated by a traffic grooming application. The MARPG problem consists in finding the maximum number of connections which can be established in a path of size $N$, where each arc has a capacity or bandwidth $C$ (grooming factor). We present a greedy algorithm to solve the problem and an explicit formula for the maximum number of requests that can be groomed. In particular, if $C=s(s+1) / 2$ and $N>s(s-1)$, an optimal solution is obtained by taking all the requests of smallest length, that is of length 1 to $s$. However this is not true in general since anomalies can exist. We give a complete analysis and the exact number of such anomalies.
\end{abstract}

Key-words: Grooming, requests, path, capacity, coloration of interval graphs

This work has been partially funded by European projects ist fet Aeolus and COST 293 GraAl, and has been done within the CRC Corso with France Telecom. 


\section{Solution optimale au problème du groupage maximum de toutes les requêtes possibles sur le chemin}

Résumé : Nous donnons une solution optimale au problème du groupage maximum de l'ensemble de toutes les requêtes possibles sur le chemin (MARPG). Ce travail est motivé par un problème de groupage de trafic. Le problème MARPG consiste à trouver le nombre maximum de connexions que l'on peut établir sur un chemin ayant $N$ sommets où chaque arc a une capacité $C$ (facteur de groupage). Nous fournissons d'une part un algorithme glouton pour résoudre ce problème et d'autre part une formule explicite donnant le nombre maximum de requêtes pouvant être groupées. En particulier, lorsque $C=s(s+1) / 2$ et $N>s(s-1)$, une solution optimale est obtenue en prenant toutes les requêtes de longueur 1 à $s$, c'est-à-dire les requêtes les plus courtes. Toutefois cette construction n'est pas optimale en général et des anomalies existent. Aussi nous donnons une analyse complète de ces anomalies et leur nombre exact.

Mots-clés : Groupage, requêtes, chemin, capacité, coloration de graphe d'intervales 


\section{Introduction}

The Maximum All Request Path Grooming (MARPG) problem that we consider in this paper is motivated by traffic grooming in an optical path network, but is of interest by itself. We are given a directed path and a number $C$ (capacity or grooming factor). A request $(i, j)$ is routed via the unique subpath from $i$ to $j$. The MARPG problem consist in finding the maximum number of simple requests (any request appears at most once) that can be routed (groomed) together such that at most $C$ requests use a given arc of the path. Said otherwise we want that the load of any arc (number of requests whose routing use this arc) does not exceed the capacity $C$ of the arc. One can also formulate the problem as : what is the maximum number of connections that can established in a network where each arc has a capacity (bandwidth) $C$ given, the network being here a path.

The MARPG problem is a particular case of the MRPG (Maximum Request Path Grooming) problem where the set of possible requests is general and not necessarily complete. As noted in $[7,9]$ there exists a polynomial time algorithm to solve the MRPG problem, and therefore to solve our problem. Indeed the MRPG problem is itself a particular case of the problem of finding a maximum $C$-colorable subgraph of an interval graph considered in $[2,10]$. In this problem we are given a set of $n$ intervals and a number $C$ and we want to find the maximum number of intervals which can be colored with one of the $C$ colors such that two intersecting intervals receive different colors.

The best known algorithm is given in [2] and has complexity $O(C+n)$. However for our grooming problem we need a closed formula and to the best of our knowledge, this does not exist in the literature. Such a formula is given in Theorem 8.

Let us now explain our motivation. In the original problem of grooming considered in [4] and [1] to each request is associated a route in the optical network and a wavelength ; each request uses at most $1 / C$ of the bandwidth or equivalently on a given arc there can be at most $C$ requests with the same wavelength. For a given set of requests the objective is to minimize the number of ADMs (Add Drop Multiplexers) used. This problem has been widely studied in the literature (see the surveys $[5,6,8,11]$ ) for various physical networks in particular for the unidirectional ring networks. In $[4,1]$ the physical network is a dipath. In [4] the problem is proved to be N-P complete for a general set of requests. In [1] the problem is modeled as a graph partition problem as follows : if the set of requests is represented by a graph $G$ the grooming problem on the path consists in partitioning the edges of $G$ into subgraphs $B_{w}=\left(V_{w}, E_{w}\right)$, such that for any arc $(i, i+1)$ of the path there are in each subgraph $B_{W}$ at most $C$ edges $(u, v)$ with $u \leq i<v$. The objective is to minimize the sum of the number of vertices of the $V_{w}$. Here $E_{W}$ corresponds to the requests with wavelength $w$ and $V_{w}$ to the number of ADM's used for this wavelength. In [1] the problem is completely solved for $C=1$ and using design theory tools for $C=2$, when the traffic is uniform All to All one, that is the request graph is complete. To solve the problem, in particular to obtain lower bounds, it was needed to know how many edges could contain a subgraph $B_{w}$ with a given number of vertices. This is exactly the MARPG problem for a dipath.

Note that for a given set of requests it is easy to compute the load of any arc $(i, i+1)$ of the path; indeed it is the number of requests $(u, v)$ with $u \leq i<v$. In particular, if we 
have all the possible requests the load of the $\operatorname{arc}(i, i+1)$ of the path $P_{N}$ with $N$ vertices is $(i+1)(n-i-1)$ and so the maximum load of an arc of the path is $\left\lceil\frac{N^{2}-\epsilon}{4}\right\rceil$ where $\epsilon=1$ if $\mathrm{N}$ is odd and 0 if $\mathrm{N}$ is even. So the problem is interesting only if $C$ is less than this value otherwise we can groom all the requests.

If $C=1$ the MARPG problem is easy to solve as an optimal solution consists in taking the $N-1$ requests of length 1 and so the maximum is $N-1$. In [1] it is proven that for $C=2$, the maximum is $\left\lfloor\frac{3 N-3}{2}\right\rfloor$ by taking all the requests of length 1 and half of that of length 2. By using duality theory, the optimum can be found for $C \leq 6$; in particular for $C=3$ (respectively $C=6$ ) the maximum is obtained by considering all the requests of length 1 and 2 (resp. 1,2 and 3), except when $C=6$ and $N=5$ where one request of length 4 is also needed.

So it was conjectured the "intuitive fact" that the optimum for the MARPG problem was obtained by taking all the requests of smallest length. However it appears that the conjecture is false (see Section 3).

In this article using matroid theory we give a "greedy" algorithm to solve optimally the MARPG problem and determine exactly for any $N$ and $C$ the optimal value of the number of requests that can be groomed.

Note that we present the problem as an oriented one as originally both the requests and the path are directed. But the problem is equivalent to that of considering symmetric requests (a symmetric request being a pair $\{u, v\}$ of nodes communicating) and undirected path.

\section{Notations and definitions}

Let $P_{N}$ be the directed path on $N$ vertices $\{0,1, \ldots, N-1\}$ with the $\operatorname{arcs} e_{i}=(i-1, i), i=$ $1, \ldots, N-1$. By definition, the request $r_{i, j}=(i, j)$, with $j>i$, loads with load 1 all the arcs of the subpath from vertex $i$ to vertex $j$. The length or size of a request $(i, j)$ is defined as $s=j-i$. We assume that the set of requests $R(N)$ is composed of all the requests of any length $s$ such that $1 \leq s \leq N-1$. The load of an $\operatorname{arc} e_{i}$ is the number of requests containing $e_{i}$ that are satisfied (or groomed) together.

The grooming factor $C$ being given, the Maximum All Request Path Grooming (MARPG) problem consists in finding the maximum number of requests $T(C, N)$ that can be groomed together such that the load of any arc of $P_{N}$ is at most $C$. One can also ask for the requests themselves that are satisfied in an optimal solution. 


\section{A false conjecture}

As said in the introduction intuitively one can think that the maximum is obtained by taking all the requests of smallest length. This is clearly true if $C=1$ as the optimum consists in taking the $N-1$ requests of length 1 . So $T(1, N)=N-1$. In [1] this has been also shown for $C=2$, the maximum being $T(2, N)=\left\lfloor\frac{3 N-3}{2}\right\rfloor$. This was also proved for $C \leq 6$, except when $C=6$ and $N=5$ where we need all requests of length 1,2,3 and 4 . Hence it was conjectured that it was true for all values of $C$.

Call $R(s)$ the set of all the requests of size $s$. Hence we have $R(N)=\bigcup_{s=1}^{N-1} R(s)$. Define

also $C_{s}=\frac{s(s+1)}{2}$. The conjecture can be stated as follows : for $C=C_{s}$, the maximum number is obtained by taking all the requests of size less than or equal to $s$. Since the number of requests of size $s$ is equal to $N-s$, the number $T_{s}(N)$ of requests of size less than or equal to $s$ is $T_{s}=s N-C_{s}$. For $C=3$, we have $T(3, N)=2 N-3$ and for $C=6$, $T(3, N)=3 N-6$ for $N \geq 6$.

However this conjecture is false as can be easily seen from the following example. Let $N=11$ and $C=10$, then $s=4$ and $T_{4}(11)=34$. But a better solution exists by deleting from the preceding solution the request $(3,7)$ of size 4 and adding the two requests of size $5,(0,5)$ and $(5,10)$, which allows to satisfy 35 requests.

Another simple example is given for $s=6, C_{s}=21$ and $N=16$. We have $T_{6}(16)=75$, but we can delete the requests of length $6,(4,10)$ and $(5,11)$, and add the 4 requests of length $7,(0,7),(7,14),(1,8)$ and $(8,15)$ leading to a solution with 77 requests.

In the rest of this paper we shall prove indeed that these numbers are optimal.

\section{Structuring the request set}

There are many ways of enumerating $R(s)$ the set of all the requests of size $s$. In the following we choose to gather the maximum number of independent requests in a vector (or set) of requests. Recall that $R(s)$ is of cardinality $N-s$ and that each request of size $s$ is of the form $(i, i+s)$ with $0 \leq i \leq N-s-1$.

For $0 \leq t<s$, let us define a request vector $R_{s, t}$ as the subset of $R(s)$ composed of the requests starting in a vertex $j \equiv t \bmod s$, that is of the form $(t+(h-1) s, t+h s)$. Hence,

$R_{s, 0}$ is the set of requests $\{(0, s),(s, 2 s), \ldots\}$ and $R_{s, t}=\{(t, t+s),(t+s, t+2 s), \ldots\}$. Note that all the requests of $R_{s, t}$ are independent and that their number of is $w(s, t)=\left\lfloor\frac{N-t-1}{s}\right\rfloor$.

Lemma 1 For any given $s$, the union of all $R_{s, t}$ for $0 \leq t \leq s-1$ is equal to $R(s)$.

Proof: The request vectors $R_{s, t}$ are obtained by gathering all the requests $(i, i+s)$ which are equal modulo $s$. Hence there exist $s$ request vectors.

In the remaining part of the paper, we shall prove that there exists a solution to the MARPG problem composed of request vectors, give an algorithm to build such a solution and use it to determine the exact value of the MARPG number.

$\mathrm{RR} \mathrm{n}^{\circ} 5627$ 


\section{An optimal solution in $R_{s, t}$}

In this section, we consider the MARPG problem from the point of view of the requests that will be satisfied in an optimal solution of the problem. The main result is that we can restrict the search for a solution to the set $R V$ of all request vectors $R_{s, t}$ where $1 \leq s \leq N-1$ and $0 \leq t \leq s-1$.

Property 2 The load induced by a set of $C$ request-vectors $R_{s, t}$, with $s \leq s_{0}$, is $C$ on all the arcs $e_{j}$ of the path $P_{N}$ such that $s_{0} \leq j \leq N-s_{0}$.

Proof: A request-vector $R_{s, t}$ loads with load 1 each $\operatorname{arc} e_{j}$ of the path $P_{N}$ such that $t+1 \leq j \leq t+s . w(s, t)$. But $t+1 \leq s \leq s_{0}$ and $t+s . w(s, t) \geq N-s \geq N-s_{0}$, proving the property.

Theorem 3 There exists an optimal solution for the MARPG problem consisting of $C$ request-vectors $R_{s, t}$.

Proof: Either there exists an optimal solution consisting of $C$ request-vectors $R_{s, t}$ and we are done. Otherwise, for any optimal solution $S$, there exists a couple $(s, t)$ such that at least one request of $R_{s, t}$ appears in $S$ and another request of $R_{s, t}$ does not appear in $S$. Let $\left(s_{0}, t_{0}\right)$ be the minimum (for the lexicographic order) couple $(s, t)$ with this property. Therefore for any $(s, t)<\left(s_{0}, t_{0}\right)$, either all the requests of $R_{s, t}$ appear in $S$ or none of them appear in $S$. Let $C_{0}$ be the number of request-vectors $R_{s, t}$, with $(s, t)<\left(s_{0}, t_{0}\right)$, appearing fully in $S$.

Consider an optimal solution $S_{0}$ such that $C_{0}$ is the greatest possible. As $S_{0}$ does not consist uniquely of request vectors, we have $C_{0}<C$. From $S_{0}$ we will build another optimal solution $S^{\prime}$, such that the request vector $R_{s_{0}, t_{0}}$ appears fully in $S$, and so for this solution we will have $C_{0}^{\prime}>C_{0}$ contradicting the maximality of $C_{0}$.

From the definition of $\left(s_{0}, t_{0}\right)$, it follows that there exist in $R_{s_{0}, t_{0}}$ two consecutive requests one appearing in $S_{0}$ and one not appearing. We will suppose that the one appearing is before (the case where it is after can be dealt similarly). Let $R_{0}=\left(t_{0}+j s_{0}, t_{0}+(j+1) s_{0}\right)$ be the request which does not appear and $\left(t_{0}+(j-1) s_{0}, t_{0}+j s_{0}\right)$ be the request appearing. Note that $j \geq 1$ and so $t_{0}+j s_{0} \geq s_{0}$.

As $S_{0}$ is optimal, we cannot add the request $R_{0}$ to $S_{0}$. Therefore among the arcs covered by the subpath associated to $R_{0}$, some of them have load $C$. Choose the one with the smallest index and call it $e^{*}$. It can be written $e^{*}=e_{t_{0}+j s_{0}+i_{0}}$, with $1 \leq i_{0} \leq s_{0}$.

Among the $C$ requests covering $e^{*}$, exactly $C_{0}$ of them belong to the $C_{0}$ request-vectors $R_{s, t}$, with $(s, t)<\left(s_{0}, t_{0}\right)$, appearing fully in $S_{0}$. Therefore there are $C-C_{0}$ requests covering $e^{*}$ belonging to some $R_{s, t}$, with $(s, t)>\left(s_{0}, t_{0}\right)$. Suppose all of them are of the form $(i, i+s)$ with $i<t_{0}+j s_{0}$, then all of them also cover the $\operatorname{arc} e_{t_{0}+j s_{0}}$. But this arc is also covered by the $C_{0}$ request-vectors $R_{s, t}$, with $(s, t)<\left(s_{0}, t_{0}\right)$, appearing fully in $S_{0}$ (indeed we can apply the property as $\left.t_{0}+j s_{0} \geq s_{0}\right)$. This arc is also covered by the request $t_{0}+(j-1) s_{0}, t_{0}+j s_{0}$ of $R_{s_{0}, t_{0}}$ before $C_{0}$. So this arc will have a load of $C+1$ which is impossible. 
Therefore among the $C-C_{0}$ requests covering $e^{*}$ and belonging to some $R_{s, t}$, with $(s, t)>\left(s_{0}, t_{0}\right)$, at least one request $R_{1}$ is of the form $(i, i+s)$, with $s \geq s_{0}$ and $t_{0}+j s_{0} \leq$ $i \leq t_{0}+j s_{0}+i_{0}-1$. As $s \geq s_{0}$, the request $R_{1}$ covers also all the arcs of $R_{0}$ after $e^{*}$ and by the minimality of $e^{*}$ the arcs of $R_{0}$ before $e^{*}$ have load at most $C-1$. So, if we delete $R_{1}$, all the $\operatorname{arcs}$ of $R_{0}$ will have a load at most $C-1$ and we can replace $R_{1}$ by $R_{0}$ without changing the maximum load $C$ obtaining therefore another optimal solution $S_{1}$ with one request more than $S_{0}$ in $R_{s_{0}, t_{0}}$. Repeating the procedure, we eventually obtain an optimal solution $S^{\prime}$ containing all the requests of the request vector $R_{s_{0}, t_{0}}$ getting the desired contradiction.

\section{Optimal greedy algorithm}

In this section we shall prove that the request vectors form a weighted matroid. Hence, the associated greedy algorithm will be optimal. Recall (see [3] for details) that a matroid is a pair $M=(S, I)$, where $S$ is a finite nonempty set and $I$, the independent set, is a nonempty family of subsets of $S$ which satisfies two properties :

1. Hereditary property : if $B \in I$ and $A \subset B$, then $A \in I$.

2. Exchange property : if $A, B \in I$ and $|A| \leq|B|$, then there is some element $x \in B-A$ such that $A \cup\{x\} \in I$.

We say that a matroid $M=(S, I)$ is weighted if there is an associated weight function that assigns a strictly positive weight $w(x)$ to each element $x \in S$.

Let $N$ be given, recall that $R$ is the set of all the requests of size not greater than $N-1$. We define $R V$ to be the set of all request vectors $R_{s, t}$, where $1 \leq s \leq N-1$ and $0 \leq t \leq s-1$. From Lemma 1, we deduce that the set of the requests of all the request vectors of size not greater than $N-1$ is equal to $R$. Hence from the point of view of the requests, $R$ and $R V$ are equal. However they differ from the structural point of view.

Theorem 3 allows us to restrict our search for an optimal solution to $R V$. For that purpose let us call $P_{C}(R V)$ the set of all subsets of $R V$ of cardinality at most $C$, where $C$ is the grooming factor. Hence an element $A$ of $P_{C}(R V)$ is composed of a set of at most $C$ request vectors $R_{s, t}$ for some values of $s$ and $t$.

Theorem 4 Given $N$ the size of a directed path and $C$ the grooming factor, the triple $\left(R V, P_{C}(R V), w\right)$ where $1 \leq s \leq N-1$ and $0 \leq t \leq s-1$ and $w(s, t)=\left\lfloor\frac{N-t-1}{s}\right\rfloor$ is a weighted matroid.

Proof: We have to prove that $P_{C}(R V)$ satisfies the two properties.

1. Hereditary property : Let $B$ be a subset of $R V$; hence $B$ is composed of at most $C$ request vectors. Any subset $A$ of $B$ is composed of less than $C$ request vectors and hence is an element of $P_{C}(R V)$. 
2. Exchange property : Let $A$ and $B$ be two subsets of $R V$ such that $|A| \leq|B|$. Hence there is at least one request vector $R_{s, t}$ in $B$ which does not belong to $A$. Since $|A|<C$, by adding $R_{s, t}$ to $A$, we get another set of at most $C$ request vectors, which is clearly in $P_{C}(R V)$.

In a weighted matroid, the independent subset that has maximum weight is called the optimal subset of the matroid. The main property of a weighted matroid is that a greedy algorithm, considering the elements of $S$ in the decreasing order of their weights, returns an optimal subset (see [3]). We deduce from this property the following theorem.

Theorem 5 Given $N$ the size of a directed path and $C$ the grooming factor, the set of the requests included in the $C$ first request vectors, ordered decreasingly by their weights, is an optimal solution to the MARPG problem.

Proof: From Theorem 3, searching an optimal solution can be restricted to searching in $R V$. In other words there exists an optimal solution for the MARPG problem which consists of $C$ request vectors.

Since $\left(R V, P_{C}(R V), w\right)$ is a weighted matroid, solving the MARPG problem in $R V$ consists in finding the maximal independent subset with maximal weight where the weight function $w(s, t)$ is the number of requests in $R_{s, t}$. The solution is given by the following greedy algorithm:

Order the set of request vectors by their decreasing weights. The greedy algorithm return the independent set of maximum weight, composed of the first $C$ request vectors and that gives a solution to the MARPG problem.

It is important to understand that the first $C$ request vectors are not composed only with the requests of smallest size. In order to illustrate this statement let us consider the example of Section 3. Take $N=11, s=4$ and $C=10$. The set of ordered request vectors is the following :

\begin{tabular}{|c|c|cc|ccc|ccc|c|}
\hline$R_{s, t}$ & $R_{1,0}$ & $R_{2,0}$ & $R_{2,1}$ & $R_{3,0}$ & $R_{3,1}$ & $R_{3,2}$ & $R_{4,0}$ & $R_{4,1}$ & $R_{4,2}$ & $R_{5,0}$ \\
\hline$w_{s, t}$ & 10 & 5 & 4 & 3 & 3 & 2 & 2 & 2 & 2 & 2 \\
\hline
\end{tabular}

Note that we do not take all the 4 request vectors of size 4 , as for $R_{4,3} w(4,3)=1$ and therefore we obtain 35 requests for the maximum number of requests that can be satisfied on a path of size 11 with a grooming factor 10 .

Remark: Theorems 4 and 5 work also for a generalization of the MARPG problem, called Maximal Multiple Request Path Grooming (MMRPG) problem. In the MMRPG problem we authorize all $R_{s, t}$ to appear $\lambda_{s, t}$ times, where $\lambda_{s, t}$ is an integer which can be zero, that is the set of requests is $\bigcup \lambda_{s, t} R_{s, t}$. Therefore, an optimal solution is obtained by taking the $C$ first admissible request vectors, ordered decreasingly by their weights. 


\section{Computation of $T(C, N)$}

We shall show that we can compute exactly the maximum number $T(C, N)$ of requests that can be groomed on a path of size $N$ with a grooming factor $C$. If we call $R V(C, N)$ the set of $C$ request vectors in an optimal solution of the MARPG problem then we have :

$$
T(C, N)=\sum_{R_{s, t} \in R V(C, N)} w(s, t)
$$

We will compute this number in two stages. First we compute the value for $C=C_{s}=$ $\frac{s(s+1)}{2}$ and then for any value of $C$. We have already seen that if the solution $R V\left(C_{s}, N\right)$ contains all (and only them) the request vectors of size at most $s$, then $T\left(C_{s}, N\right)=T_{s}=$ $s N-C_{s}$. But this is not true in general. Hence we shall call anomalies, the number of requests that can be satisfied in excess of $T_{s}$. This is this number that we have to compute.

First let us note the following property.

Property 6 For fixed value of $t, w(s, t)$ is a decreasing function of $s$, i.e., for all $s, w(s+$ $1, t) \leq w(s, t)$. For fixed value of $s, w(s, t)$ is a decreasing function of $t$, i.e., for all $t$, $w(s, t+1) \leq w(s, t)$.

Lemma 7 Given $N, s \geq 1$ and $C_{s}=\frac{s(s+1)}{2}$, let $N=q s+r$ where $0 \leq r \leq s-1 ; r=a q+\alpha$ where $0 \leq \alpha \leq a-1$ and $s-r=b(q+1)+\beta$ where $0 \leq \beta \leq b-1$. The number of requests of an optimal solution for the MARPG problem is

$$
T\left(C_{s}, N\right)=T_{s}+\min \left(A_{s}, B_{s}\right)
$$

where $T_{s}=s N-C_{s}, A_{s}=a r-\frac{a(a+1)}{2} q$, and $B_{s}=(b+1)(s-r)-\frac{b(b+1)}{2}(q+1)$.

Proof: Among the $s$ request vectors of $R(s)$, we deduce from the decomposition $N=q s+r$ that there are $r$ vectors of cardinality $q$ (since $w(s, t)=q$ ) and $s-r$ vectors of cardinality $q-1$. Similarly, among the $s+k$ request vectors of $R(s+k)$, there are $r-k q$ vectors of cardinality $q$ and $s-r+k q$ vectors of cardinality $q-1$. This is true for all values of $k$ such that $r-k q \geq 0$, hence for $k \leq a$. Call $A_{s}$ the number of request vectors of cardinality $q$ in $R(s+k)$ for $1 \leq k \leq a$. We have : $A_{s}=a r-\frac{a(a+1)}{2} q$.

Moreover, among the $s-h$ request vectors of $R(s-h)$ there are $s-h-r-h q=$ $s-r-h(q+1)$ vectors of cardinality $q-1$ for $0 \leq h \leq b$. Call $B_{s}$ the number of request vectors of cardinality $q-1$ in $R(s-h)$ for $0 \leq h \leq b$. We have : $B_{s}=(b+1)(s-r)-\frac{b(b+1)}{2}(q+1)$.

By the definition of $A_{s}$, we deduce that we will have some anomalies if $A_{s} \geq 0$. This means that in the request vectors of $R(s+k)$, for $1 \leq k \leq a$, we have some of them with one request more than those in $R(s-h), 0 \leq h \leq b$. In the non decreasing order of weights the $B_{s}$ request vectors of cardinality $q-1$ will appear after the $A_{s}$ request vectors of cardinality $q$ and so we should replace them. The maximum number of such replacements is $\min \left(A_{s}, B_{s}\right)$. 
We can now prove the main result of the paper. For that remark that any positive number $C$ can decomposed into $C=C_{s}-d$ with $0 \leq d \leq s-1$.

Theorem 8 Given $N$ and $C$, let $C=C_{s}-d$ with $0 \leq d \leq s-1 ; N=q s+r$ where $0 \leq r \leq s-1 ; r=a q+\alpha$ where $0 \leq \alpha \leq a-1$ and $s-r=b(q+1)+\beta$ where $0 \leq \beta \leq b-1$. Then the number of requests of an optimal solution for the MARPG problem is

$$
T(C, N)=T_{s}-d q+\min \left(A_{s}+d, B_{s}\right)
$$

where $T_{s}=s N-C_{s}, C_{s}=\frac{s(s+1)}{2}, A_{s}=a r-\frac{a(a+1)}{2} q$ and $B_{s}=(b+1)(s-r)-\frac{b(b+1)}{2}(q+1)$.

Proof: Starting from a solution of the MARPG problem with $C=C_{s}$, we can build a solution for $C=C_{s}-d$ by removing the last $d$ request vectors from the solution. Among these vectors assume that $d_{1}$ are of cardinality $q$ and $d_{2}$ of cardinality $q-1$ where $d_{1}+d_{2}=d$. From the definition of $A_{s}$ and $B_{s}$ we deduce that:

$$
T(C, N)=T_{s}-d_{1} q-d_{2}(q-1)+\min \left(A_{s}+d_{1}, B_{s}-d_{2}\right)
$$

Hence :

$$
T(C, N)=T_{s}-d q+d_{2}+\min \left(A_{s}+d_{1}, B_{s}-d_{2}\right)
$$

which leads to the formula and concludes the proof.

We show in Figure 1 the number of anomalies (gap between $T(C, N)$ and the value of the false conjecture) for grooming factor $C=192$ and $C=256$ (effective values for SONET networks).
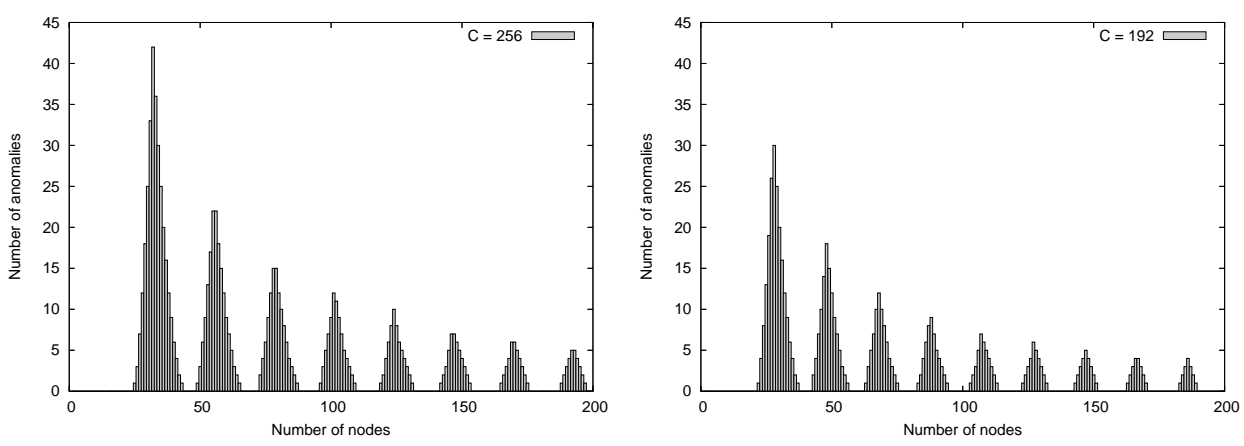

Figure 1: Number of anomalies for grooming factor $C=256$ and 192 . 
We now give some complementary properties on the solution of the MARPG problem.

Corollary 9 Let $N, s$ and $C=C_{s}$ be given, a necessary and sufficient condition for the absence of anomalies is : $(N \bmod s) \leq\left\lfloor\frac{N}{s}\right\rfloor$, or equivalently $N=u s+v(s+1)$, with $u$ and $v$ positive integers.

Proof: Let $N=q s+r$ with $0 \leq r \leq s-1$, that is $r \equiv N \bmod s$ and $q=\lfloor N / s\rfloor$. From the definition of $B_{s}$, it can be easily seen that $B_{s} \geq s-r>0$. Hence in order to have no anomalies, a necessary and sufficient condition is that $A_{s}=0$. This condition is equivalent to $r \leq q$ which can be also written as $N=(q-r) s+r(s+1)$.

Corollary 10 Let $s$ and $C=C_{s}$ be given. If $N>s(s-1)$, then there is no anomalies and $T\left(C_{s}, N\right)=T_{s}$.

\section{Conclusion}

In this article we have completely solve the problem of determining the maximum number of requests which can be groomed in a path with a capacity $C$ on each arc. We have shown furthermore that optimal solutions were obtained with a greedy algorithm. It will be interesting to consider the same problem for other networks. Note that for unidirectional rings this problem is easy as the solution is given by considering all the requests of smallest length.

\section{References}

[1] J-C. Bermond, L. Braud, and D. Coudert. Traffic grooming on the path. In 12th International Colloquium on Structural Information and Communication Complexity SIROCCO, pages 34-48, Le Mont Saint-Michel, France, May 24-26 2005. LNCS 3499.

[2] M. C. Calisle and E. L. Lloyd. On the $k$-coloring of intervals. Discrete Applied Mathematics, 59(3):225-235, 1995.

[3] T. Cormen, C. Leiserson, and R. Rivest. Introduction to Algorithms. The MIT Press, 1990.

[4] R. Dutta, S. Huang, and N. Rouskas. On optimal traffic grooming in elemental network topologies. In Opticomm, pages 13-24, Dallas, October 2003.

[5] R. Dutta and N. Rouskas. A survey of virtual topology design algorithms for wavelength routed optical networks. Optical Networks, 1(1):73-89, January 2000.

[6] R. Dutta and N. Rouskas. Traffic grooming in WDM networks: Past and future. IEEE Network, 16(6):46-56, November/December 2002.

$\mathrm{RR} \mathrm{n}^{\circ} 5627$ 
[7] T. Erlebach and K. Jansen. Maximizing the number of connections in optical tree network. In Proceedings of the Ninth Annual International Symposium on Algorithms and Computation (ISAAC'98), pages 179-188. Springer Verlag, LNCS 1533, 1998.

[8] E. Modiano and P. Lin. Traffic grooming in WDM networks. IEEE Communications Magazine, 39(7):124-129, July 2001.

[9] P. Winkler and L. Zhang. Wavelength assignment and generalized interval graph colorin. In ACM-SIAM Symposium on Discrete Algorithms (SODA), pages 830-831 (extended abstract, 2003.

[10] M. Yannakakis and F. Gavril. The maximum $k$-colorable subgraph problem for chordal graphs. Information Processing Letters, 24(2):133-137, January 1987.

[11] K. Zhu and B. Mukherjee. A review of traffic grooming in WDM optical networks: Architectures and challenges. Optical Networks Magazine, 4(2):55-64, March/April 2003. 


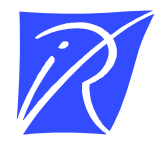

\section{Unité de recherche INRIA Sophia Antipolis 2004, route des Lucioles - BP 93 - 06902 Sophia Antipolis Cedex (France)}

Unité de recherche INRIA Futurs : Parc Club Orsay Université - ZAC des Vignes 4, rue Jacques Monod - 91893 ORSAY Cedex (France)

Unité de recherche INRIA Lorraine : LORIA, Technopôle de Nancy-Brabois - Campus scientifique 615, rue du Jardin Botanique - BP 101 - 54602 Villers-lès-Nancy Cedex (France)

Unité de recherche INRIA Rennes : IRISA, Campus universitaire de Beaulieu - 35042 Rennes Cedex (France)

Unité de recherche INRIA Rhône-Alpes : 655, avenue de l'Europe - 38334 Montbonnot Saint-Ismier (France)

Unité de recherche INRIA Rocquencourt : Domaine de Voluceau - Rocquencourt - BP 105 - 78153 Le Chesnay Cedex (France)

INRIA - Domaine de Voluceau - Rocquencourt, BP 105 - 78153 Le Chesnay Cedex (France)

http://www.inria.fr

ISSN 0249-6399 